\title{
Lower hybrid turbulence and ponderomotive force effects in space plasmas subjected to large-amplitude low- frequency waves
}

\author{
G. V. Khazanov and T. E. Moore \\ Space Sciences Laboratory, Huntsville, Alabama \\ E. N. Krivorutsky, and J. L. Horwitz \\ Department of Physics and Center for Space Plasma and Aeronomic Research, The University of Alabama in \\ Huntsville
}

\author{
M. W. Liemohn \\ Space Physics Research Laboratory, University of Michigan, Ann Arbor, Michigan
}

\begin{abstract}
It is demonstrated that large-amplitude low-frequency waves (LFW) can generate lower hybrid waves (LHW) in the auroral zone and ring current region. The LHW could then heat the ions. The ion energization due to the LHW may be comparable with that produced by the ponderomotive force of the LFW.
\end{abstract}

\section{Introduction}

Wave-particle interactions are of crucial importance to magnetospheric and ionospheric plasma behavior. Space plasmas support a wide variety of large-amplitude waves. For example, in the region of overlap between the ring current and the outer plasmasphere numerous observations have shown large-amplitude waves with frequencies below the local proton cyclotron frequency [McPherron et al., 1972; Taylor et al., 1975; Kintner and Gurnett, 1977; Young et al., 1981; Perraut, 1982; Roux et al., 1982; Fraser, 1985; Labelle et al., 1988]. These observations indicate the presence of waves with both left-hand polarization (Alfven waves), and right-hand polarization (fast magnetosonic waves). It is known that the generation of ion cyclotron waves may occur as a result of an instability in the high energy anisotropic component of the ring current during interaction with the plasmasphere [Cornwall, 1965, 1966; Kennel and Petschek, 1966; Liemohn, 1967; McKean et al., 1994]. In the auroral region, simultaneous occurrences of upward-flowing ions and field-aligned electrons have been observed by the Viking satellite. The occurrence is strongly correlated with large amplitude low-frequency fluctuations of the electric field [Hultqvist et al., 1988; Lundin et al., 1990]. Large-amplitude shear Alfvén waves were also observed by sounding rockets [Boehm et al., 1990] in the auroral ionosphere.

When such low-frequency waves (LFW) propagate in a plasma, a ponderomotive force is produced which may lead to significant effects at the plasma. Boehm et al. [1990] sug-

Copyright 1996 by the American Geophysical Union.

Paper number 96GL00844

0094-8534/96/96GL-00844\$05.00 gested that this ponderomotive force can be the cause of significant density perturbations. Allan [1993] has investigated mass transport caused by the ponderomotive force of hydrodynamic waves in the middle magnetosphere. It was also suggested that significant plasma energization could occur in these regions. $L i$ and Temerin [1993] have studied the ion energization by the ponderomotive force of large-amplitude Alfvén waves $\left(\mathrm{E}>100 \mathrm{mV} / \mathrm{m} ; \omega<\Omega_{1}\right)$ in the auroral zone.

It is well known [eg. Akhiezer et al., 1975] that in the field of a L.FW, electrons and ions move with different velocities. A significant ion-electron relative velocity may lead to a current instability which may produce, for example, lower hybrid waves (LHW). The purpose of this paper is to consider the possibility of such L.HW generation by large-amplitude LFW in a space plasma and to compare the effects of LHW and the ponderomotive force in the processes of heating and acceleration in the plasma.

Below we discuss the possibility of LHW generation in the ring current region and in the auroral zone and mechanisms for LH instability saturation. The LHW power level and the resulting plasma energization due to the $\mathrm{LH}$ turbulence are estimated. The plasma energization due to $\mathrm{LH}$ turbulence is compared with that due to the ponderomotive force.

\section{Analysis}

\section{Lower Hybrid Current Instability Due to Low- Frequency Waves}

If a plasma is subjected to a variable electric field $\vec{E}$ (for instance, the electric field of an Alfvén or fast magnetosonic wave, which has a component at a right angle to the external magnetic field $\vec{B}_{o}$ ), the electrons and ions will acquire different velocities $\vec{u}_{e}$ and $\vec{u}_{i}$ under the action of that field. If the frequency $\omega_{0}$ of the field is considerably lower than the electron cyclotron frequency, the electron and ion velocities will be given by [eg., Akhiezer et al., 1975]:

$$
\begin{aligned}
& \vec{u}_{e}=c \frac{\vec{E} \times \vec{B}_{o}}{B_{o}^{2}}-i \frac{e(\vec{E} \cdot \vec{b}) \vec{b}}{m_{e} \omega_{0}} \\
& \vec{u}_{i}=\frac{e}{m_{i}\left(\omega_{B i}^{2}-\omega_{o}^{2}\right)}\left\{\omega_{B i}(\vec{E} \times \vec{b})+i \omega_{o}((\vec{E} \times \vec{b}) \times \vec{b})\right\}
\end{aligned}
$$

where $\vec{b}$ is a unit vector in the direction of the external mag- 
netic field and $\omega_{B i}$ is the ion cyclotron frequency. In a lowfrequency field the component of the parallel electric field is small, and the perpendicular drift velocities of the electrons and ions may be comparable to, or even larger than, the parallel electron velocity. We will restrict ourselves to the case when the parallel electric field and parallel electron velocity may be neglected.

In a coondinate system where the magnetic field $\vec{B}_{o}$ is parallel to the $\mathrm{z}$ axis and a low-frequency $\left(\omega_{0}<<\omega_{\mathrm{Bi}}\right)$ electric field $\vec{E}=\vec{E}_{o} e^{-i \omega_{o} t}$ is parallel to the $\mathrm{x}$ axis, the real part of the relative velocity of the ions with respect to the electrons, $\vec{u}=\vec{u}_{i}-\vec{u}_{e}$, is :

$$
\operatorname{Re} u_{x} \approx-\frac{e \omega_{o}}{m_{i} \omega_{B i}^{2}} E_{o} \sin \omega_{o} t
$$

Here we took into account the inequality $\omega_{0} \ll \omega_{B i}$ and neglected terms proportional to $\mathrm{m}_{\mathrm{e}} / \mathrm{m}_{\mathrm{i}}$.

For some cases, the relative velocity (2) can be larger than the ion thermal velocity $\mathrm{v}_{T i}=\sqrt{T_{i} / m_{i}}$. It may lead to the occurrence of the current instability even for the relatively small electric field of low-frequency oscillations. The inequality $u>v_{\mathrm{Ti}}$ leads to:

$$
\frac{E_{o}^{2}}{8 \pi n T} \geq \frac{\omega_{B i}^{4}}{\omega_{o}^{2} \omega_{p i}^{2}}
$$

where $\mathbf{n}$ and $\mathbf{T}$ are density and temperature of the plasma, respectively; $\omega_{p i}$ is the ion plasma frequency.

For plasmas in the auroral region and near the plasmapause, condition (3) requires a LFW electric field greater than that observed on average, but one that is not uncommon. Inequality (3) appears to be satisfied in observations reported by Taylor et al. [1975] and Young et al. [1981] for the ring current region ( $\mathrm{L}=4-5$ ). It was found that $B^{2} / B_{o}^{2}=5 \cdot 10^{-4}$ (B is the magnetic field of the Alfven wave, $B_{0}$ is the ambient field), $\omega_{0}=1-3 \mathrm{~s}^{-1}, \mathrm{n}_{\text {cold }}=30 \mathrm{~cm}^{-3}, \mathrm{n}_{\text {hot }}=1 \mathrm{~cm}^{-3}, \mathrm{~T}_{\text {cold }}=0.5 \mathrm{eV}, \mathrm{T}_{\text {hot }}=(5-$ 50) $\mathrm{keV}$. If the plasma is isothermal, $T_{e} \approx T_{i}$, and $u \approx 1.3 \mathrm{v}_{\mathrm{Ti}}$, then the flux instability for LH oscillation occurs [Akhiezer et al., 1975]. So, inequality (3) is satisfied if $B^{2} / B_{o}^{2} \geq 10^{-4}$, and therefore for this plasma. For the auroral acceleration region, the example considered by $L i$ and Temerin [1993], in which $\mathrm{E}=200 \mathrm{mV} / \mathrm{m} ; \omega_{\mathrm{o}}=6 \mathrm{~s}^{-1} ; \mathrm{n}_{\mathrm{o}}=4 \mathrm{~cm}^{-3} ; \mathrm{n}_{\mathrm{H}}=6 \mathrm{~cm}^{-3} ; \mathrm{T}_{\mathrm{i}}=0.5 \mathrm{eV}$, satisfies inequality (3) above $4500 \mathrm{~km}$. The ion-sound instability occurs if $u>2 v_{T_{1}}$ and the existence of the ion-sound oscillation is possible $\left(T_{e} \gg T_{i}\right)$. In a plasma with hot ions and cold electrons, $T_{e}<<T_{i}$, and $u \leq \mathrm{v}_{\mathrm{T}_{i}}$, the relative motion of ions with respect to electrons across the magnetic field can lead to the excitation of electron sound waves [eg. Akhiezer et al., 1975]. Also, depending on $u, \omega_{0}$ and the plasma parameters, the excitation of other types of plasma oscillations is possible, even for $u \ll<v_{T_{i}}$ [Gamayunov et al., 1993].

Consider an isothermal plasma $T_{e} \approx T_{i}$ for which low-frequency oscillations lead to the inequality $v_{T_{i}} \ll u<<v_{T_{e}}$. In this case, there occurs an aperiodic flux instability of LH oscillations [eg. Akhiezer et al., 1975]:

$$
\operatorname{Re} \omega-\operatorname{Im} \omega \equiv \gamma_{p} \sim k_{\perp} u_{\perp}=\frac{\omega_{p i} \omega_{B e}}{\sqrt{\omega_{p e}^{2}+\omega_{B e}^{2}}}=\omega_{L H}
$$

\section{Estimation of Lower ,Hybrid Energy Density}

Let us estimate the energy density of the LH oscillations. Generation of these waves takes place in the region of angles where $\cos ^{2} \theta \sim m_{e} / m_{i}$ ( $\theta$ is the angle between the wave vector of LH oscillations $\vec{k}$ and the magnetic field $\vec{B}_{o}$ ). The main nonlinear process which leads to saturation of the LH instability for such angles is induced scattering of $\mathrm{LH}$ oscillations from electrons. The characteristic damping rate for the induced scattering is [Musher et al., 1978]:

$$
\gamma_{s}^{e}=\frac{\omega_{p e}^{4} \omega_{B_{e}}^{2}}{\left(\omega_{p e}^{2}+\omega_{B e}^{2}\right)^{2} \omega_{L H}} \frac{W}{n T}
$$

where $\mathrm{W}$ is the energy density of the LH oscillations and $\omega_{B e}$ is the electron cyclotron frequency.

From the balance of the generation rate (4) and the damping rate (5) (i.e., the stationary condition) we can estimate the energy density of quasi-stable LHW as:

$$
\frac{W}{n T} \sim \frac{m_{e}}{m_{i}}\left(1+\frac{\omega_{B e}^{2}}{\omega_{p e}^{2}}\right)
$$

At these energy densities the plasma should not be considered as weakly turbulent, since the condition

$$
\frac{W}{n T} \geq \frac{m_{e}}{m_{i}}\left(1+\frac{\omega_{B e}^{2}}{\omega_{p e}^{2}}\right) k^{2} R^{2}\left\{\begin{array}{l}
R^{2} \sim \frac{\mathrm{v}_{\mathrm{T}_{\mathrm{e}}}^{2}}{\omega_{p_{e}}^{2}} ;\left(\omega_{B e} \gg \omega_{p e}\right) \\
R^{2} \sim \frac{\mathrm{v}_{\mathrm{T}_{e}}^{2}}{\omega_{B_{e}}^{2}} ;\left(\omega_{B e} \ll<\omega_{p e}\right)
\end{array}\right\}
$$

exists, and at this level, there occurs a modulation instability of LHW which leads to the formation of cavitons [Musher and Sturman, 1975]. Here, $k$ is the characteristic value of the wave number from the spectrum of these waves. Using (4) and $k \sim \omega_{L H} / u$, we can present condition (7) in the form:

$$
\begin{aligned}
\frac{W}{n T} & \geq \frac{\mathrm{v}_{\mathrm{Ti}}^{2}}{u^{2}} \frac{m_{e}}{m_{i}} ; \quad\left(\omega_{B e} \ll \omega_{p e}\right) \\
\frac{W}{n T} & \geq \frac{\mathrm{v}_{\mathrm{T} i}^{2}}{u^{2}} \frac{m_{e}}{m_{i}} \frac{\omega_{B e}^{2}}{\omega_{p e}^{2}} ; \quad\left(\omega_{B e}^{2} \gg \omega_{p e}^{2}\right)
\end{aligned}
$$

Taking into account the inequality $u>v_{T_{3}}$, it is clear from (6) and (8) that condition (7) is fulfilled. Let us evaluate the velocity of the energy deposition $Q$ into the plasma due to the LH turbulence. In this case, according to Musher et al. [1986], we have:

$$
\frac{Q}{n T} \approx \omega_{L H} \frac{W}{n T}-\frac{m_{e}}{m_{i}} \omega_{L H}\left(1+\frac{\omega_{B e}^{2}}{\omega_{p e}^{2}}\right)
$$

This means that excitation of LHW by a LFW can lead to the appearance of an additional channel of energy transfer from, for example, Alfvén or fast magnetosonic waves, to the particles. This process can influence the formation of the plasma distribution function through particle acceleration in its "tail" during the collapse. This influences the transport processes, since additional collisions of particles with cavitons will occur [Musher et al., 1986]. As is well known, the strong LH turbulence contributes to transverse ion beating [Chang and Coppi, 1981].

The process described above can develop in the following way. The plasma temperature will increase until the thermal ion speed reaches the threshold of excitation for LHW. When $u \approx v_{\mathrm{T}_{1}}$, relation (3) will not be satisfied anymore and the gen- 
eration of the current instability will be destroyed. At that time the ion energy is approximately

$$
\varepsilon_{L H}=\frac{m_{i} u^{2}}{2} \approx \frac{\omega_{p i}^{2} \omega_{o}^{2}}{\omega_{B_{t}}^{4}} \frac{E_{o}^{2}}{16 \pi n}
$$

Expression (9) allows us to estimate the effective frequency of collisions, $v_{\text {eff }}$, of plasma particles with LH cavitons. If we introduce $v_{e f f}$ with the expression $Q=v_{e f f} n T$ and compare it with (9), we obtain:

$$
v_{e f f} \approx \omega_{L H} \frac{m_{e}}{m_{i}}\left(1+\frac{\omega_{B e}^{2}}{\omega_{p e}^{2}}\right)
$$

This frequency may be greater than the collisional frequency for ions in the magnetosphere. For example, for the ring current region $(\mathrm{L}=4) \quad v \approx 3 \cdot 10^{-4} s^{-1} ; \quad \omega_{L H} \approx 250 \mathrm{~s}^{-1} ;$ and $v_{\text {eff }} \approx 0.1 \mathrm{~s}^{-1}$.

In a multicomponent plasma where species have different drift velocities, the ion $\mathrm{LH}$ caviton collisions may lead to an effective frictional force. Thus, the LH turbulence, which occurs in a field of low-frequency oscillations, will influence the plasma energy density and momentum.

\section{Comparison of Lower Hybrid Turbulence and Ponderomotive Force Energization}

Let us compare the effects of $\mathrm{LH}$ turbulence and the ponderomotive force on the processes of acceleration and heating in the plasma. The expression for the ponderomotive force $\vec{F}_{p}$ which affects particles in a cold, magnetized plasma can be written [ $\mathrm{Li}$ and Temerin, 1993]:

$$
\vec{F}_{p}=\frac{e^{2}}{4 m} \nabla\left(\frac{E_{o \perp}^{2}}{\omega_{B}^{2}-\omega_{o}^{2}}-\frac{E_{o l l}^{2}}{\omega_{o}^{2}}\right)
$$

For the ions, in the case $\omega_{B_{t}}^{2} \gg \omega_{0}^{2}$, we have:

$$
\vec{F}_{p}=\frac{e^{2}}{4 m_{i}} \nabla\left(\frac{E_{o \perp}^{2}}{\omega_{B i}^{2}}\right)
$$

Let us now compare the effects of the energization of the plasma due to the ponderomotive force and $\mathrm{LH}$ turbulence. The ponderomotive force is a potential force. In this case, the energy gain over length $\ell$ is simply determined by the potential drop $\Psi_{p}=-\left(e^{2} E_{o}^{2}\right) /\left(4 m_{i} \omega_{B_{i}}^{2}\right)$ on this scale. If the characteristic length of the potential drop is $L$, and we neglect the initial energy of the particle, the obtained ion energy can be written as:

$$
\varepsilon_{p} \approx \frac{e^{2}}{4 m_{i}} \frac{E_{o}^{2}}{\omega_{B_{i}}^{2}} \frac{\ell}{L}=\frac{\omega_{p i}^{2}}{\omega_{B i}^{2}} \frac{E_{o}^{2}}{16 \pi n} \frac{\ell}{L}
$$

Therefore, the ion energization depends on the magnetic field, the scale length of the energy density of the low-frequency oscillation, and the length of the region of acceleration.

The heating due to the LH turbulence (10) is determined by the relation between the frequency of the LH oscillation and the cyclotron frequency of the ions and will take place in all regions where LFW exist and where the condition of the generation of LH oscillations, $u \geq v_{T_{1}}$, is satisfied.

The comparison of $\varepsilon_{p}$ and $\varepsilon_{L H}$ shows that the effectiveness of these processes depends on the relation between the parameters $\ell / L$ and $\omega_{o}^{2} / \omega_{B_{i}}^{2}$. The contribution of the LH tur- bulence to the ion heating will be the predominant process during the first stages of the plasma acceleration until $\ell \ll L$ and $\ell / L<\omega_{o}^{2} / \omega_{B_{i}}^{2}$.

\section{Discussion and Conclusions}

Let us examine some of the above-mentioned relations for the region of the ring current and estimate the time scale for energy deposition from Alfvén oscillations, $\left(\gamma_{p}^{A}\right)^{-1}$, due to the generation of the LH oscillation current. In this region, large-amplitude Alfvén waves generated by anisotropic protons were observed by Taylor et al. [1975] and Young et al. [1981]. Due to exponentially weak Alfvén wave damping and regularity of the magnetic field in the equatorial region along magnetic lines, the ponderomotive potential drop $\Psi_{p} \sim E_{o}^{2} / B_{o}^{2}$ and the ion energy change under the influence of the ponderomotive force (17) are negligible. However, as discussed by Gamayunov et al. [1992], the excitation of LH turbulence by Alfvén waves can play an important role in the saturation of the Alfven oscillations, limiting their energy density level. For steady-state conditions, the following relation can be used:

$$
\gamma_{p}^{A} W^{A}=\gamma_{p} W
$$

where $\gamma_{p}^{A}$ is the linear growth rate of Alfvén wave; and $W^{A}$ and $W$ are the energy densities for Alfvén and LHW, respectively. The linear growth rate of Alfven wave generation by anisotropic protons of the ring current during the cyclotron interaction has the form [Kennel and Petschek, 1966]:

$$
\gamma_{p}^{A}=\frac{A \sqrt{\pi}}{2} \mu \frac{\omega_{B_{i}}^{3}}{k_{11} V_{T_{k}} \omega_{A}},
$$

where $A$ is the temperature anisotropy of the ring current protons and $\mu$ is the relative concentration of anisotropic particles. For $\mu \sim 10^{-2}$ and $V_{A} / V_{T_{h}} \sim 10^{-1}\left(V_{T_{h}}\right.$ is the thermal speed of hot protons), we have $\gamma_{P}^{A} \sim 10^{-1} \omega_{A}$. Using (4) and (6) for $\mathrm{L} 5, \mathrm{n}_{\mathrm{hot}}=1 \mathrm{~cm}^{-3}, \mathrm{n}_{\text {cold }}=10^{2} \mathrm{~cm}^{-3}, \mathrm{~T}_{\mathrm{hot}}=2.510^{4} \mathrm{eV}$ and $\mathrm{T}_{\text {cold }}=0.5 \mathrm{eV}$ we can estimate $\mathrm{W}^{\mathrm{A}}=\mathrm{B}^{2} / 8 \pi$. The obtained result is in good agreement with experimental data [Taylor et al., 1975; Young et al., 1981]: $B^{2} / B_{o}^{2} \sim 10^{-4}$ (B is the magnetic field of the wave, $B_{0}$ is the ambient field).

Thus, the large amplitude Alfvén waves can generate LH oscillations in the ring current region. This process may also limit the growth of Alfven oscillations here. LHW have apparently been observed by Dynamics Explorer 1 near the geomagnetic equator [Olsen et al, 1987]. Evaluations that could be made on the basis of the data of Olsen indicate a fairly high level of energy density of the oscillations, $W / n T$ $10^{-6}-10^{-7}$ ( $W$ is the energy density of LHW; $n$ and $T$ are the density and temperature of the plasma).

From the above discussion, it follows that in the presence of the large-amplitude LFW

$$
\frac{E_{o}^{2}}{8 \pi n T} \geq \frac{\omega_{B i}^{4}}{\omega_{o}^{2} \omega_{p i}^{2}}
$$

LHW may be generated. Such conditions are fulfilled in some cases in the auroral zone and in the ring current region $\mathrm{L}-5$. The LHW contribute to ion and electron heating. The ion energy due to this process will approach 


$$
\frac{m_{i} u^{2}}{2}=\frac{\omega_{p i}^{2} \omega_{o}^{2}}{\omega_{B i}^{4}} \frac{E_{o}^{2}}{16 \pi n}
$$

This energy is comparable with energization due to the ponderomotive force of the LFW. As may be found from (6), the energy density of the LHW electric field is of order $|E|^{2} / n T \sim V_{A}^{2} / c^{2}$ ( $V_{A}$ is the Alfvén velocity). The LH turbulence generated by Alfvén waves in the ring current region may lead to saturation of Alfvén oscillations [Gamayunov et al., 1992].

So it may be supposed that LHW excitation may lead to the appearance of an additional channel of energy transfer from LFW to the particles. Such energy transfer may influence the formation of the distribution functions of the plasma species (transverse heating, particle acceleration within the "tails" of the distribution functions).

Analysis of the effects of the pondermotive force of largeamplitude LFW must take into account the possibility of LHW generation and effects connected with this.

It may be concluded from the expressions of (2) and (3) that the relative ion velocity in the electric field of LFW increases with ion mass. The reason is that the relative velocity of the various ions is caused by the different inertia of ions and electrons in the oscillating electric field. The magnetosphere and ionosphere have multicomponent plasmas, and it can be supposed that LHW can be excited by LFW with amplitudes less than that needed for a proton-electron plasma. The minimum energy density of the LFW electric field can be estimated for our case as $-\left(m_{H} / m_{O}\right)^{2}$ times less than for a purely hydrogen plasma, where $m_{H}$ and $m_{O}$ are the masses of hydrogen and oxygen, respectively.

Let us note, in conclusion, that the present results are of a qualitative nature. To clarify the authenticity of the suggested physical mechanisms, a detailed quantitative description is required as well as a detailed comparison of theoretical results with experimental data.

Acknowledgments. This work was supported at the University of Alabama in Huntsville by NSF grants ATM-9523699, ATM-9301024 and ATM-9402310. One of us, G. V. Khazanov, held a National Research Council-Marshall Space Flight Center Senior Research Associateship while this work was performed.

\section{References}

Akheizer A.I. (ed.), Plasma electrodynamics, Pergamon Press, New York, 1975

Allan, W., Plasma energization by the ponderomotive force of magnetospheric standing Alfvén waves, J. Geophys. Res., 98, 11383, 1993.

Boehm, M.H., C. W. Carlson, J. P. McFadden, J. H. Clemmons, and F. S. Mozer, High-resolution sounding rocket observation of large-amplitude Alfvén waves, J. Geophys. Res., 95, 12157, 1990.

Chang, T., and B. Coppi, Lower hybrid accelaration and ion evolution in the subauroral region, Geophys. Res. Lett., 8, 1253, 1981.

Cornwall, J. M., Cyclotron instabilities and electromagnetıc emission in the ultra low frequency and very low frequency ranges, J. Geophys. Res., 70, 61, 1965.

Cornwall, J. M., Micropulsations and the outer radiation zone, $J$. Geophys. Res., 71, 2185, 1966.

Fraser, B. J., Observations of ion cyclotron waves near synchronous orbit and on the ground, Space Sci. Rev., 42, 357, 1985.
Gamayunov, K. V., E. N. Krivorutsky, A. A. Veryaev, and G. V. Khazanov, Saturation of Alf vén oscillations in the ring current regıon due to generation of lower hybrid waves, Planetary and Space Science, 40, 477, 1992.

Gamayunov, K. V., G. V. Khazanov, E. N. Krivorutsky, and A. A. Veryaev, Parametric excitation of high frequency electromagnetic waves by the lower frequency dipole pumping, Physics of Fluids B: Plasma Physics, 5, 92-103, 1993.

Hultqvist, B., R. Lundin, K. Stasiewicz, L. Block, P.-A. Lindqvsit, G. Gustafesson, H. Koskınen, A. Bahnsen, T. A. Potemra, and L. J. Zanetti, Simultaneous observation of upward moving field-alıgned energetuc electrons and ions on auroral zone field lines, $J$. Geophys. Res., 93, 9765, 1988.

Kennel, C. F., and H. E. Petschek, Limit on stably trapped particle fluxes, J. Geophys. Res., 71, 1, 1966.

Kintner, P. M., and D. A. Gurnett, Observations of ion cyclotron waves within plasmasphere by Hawkeye 1, J. Geophys. Res., 82, 2314, 1977.

LaBelle, J., R. A. Treumann, W. Baumjohann, G. Haerendel, N. Sckopke, G. Paschmann, and Lühr, H., The duskside plasmapause/ring current interface: Convection and plasma wave observations, J. Geophys. Res., 93, 2573, 1988.

$\mathrm{Li}, \mathrm{X}$., and M. Temerin, Ponderomotive effects on ion acceleration in the auroral zone, Geophys. Res. Lett., 20, 13, 1993.

Liemohn, H. B., Cyclotron resonance amplification of VLF and ULF whistlers, J.Geophys. Res., 39, 1967.

Lundin, R., G. Gustafesson, A. I. Eriksson, and G. Marklund, On the importance of high-altitude low-frequency electric fluctuations for the escape of ionospheric ions, J. Geophys. Res., 95, 5905, 1990.

McKean, M. E., D. Winske, and P. P. Gary, Two-dimensional simulations of ion anisotropy instabilities in the magnetosheath, $J$. Geophys. Res., 94, 11141, 1994.

McPherron, R. L., C. T. Russell, and P. J. Coleman, Fluctuating magnetic fields in the magnetosphere, 2, ULF waves, Space Sci. Rev., 13, 411, 1972.

Musher, S. L., and B. I. Sturman, Collapse of plasma waves near the lower hybrid resonance, JETP Lett., 22, 265, 1975.

Musher, S. L., A. M. Rubenchik, and B. I. Sturman, Collective effects associated with low hybrid heatung of plasma, Plasma Physics, 20, 1131, 1978.

Musher, S. L., A. M. Rubenchik, and I. Ya., Shapiro, Nonlinear effects in the propagation of an ion beam across a magnetic field, Sov. Phys. JETP, 63, 519, 1986.

Olsen, R. C., C. R. Chappell, D. L. Gallagher, J. L. Green, and R. R. Andersen, Plasma observations at the magnetic equator, J. Geophys. Res., 92, 2385, 1987.

Perraut, S., Wave-particle interactions in the ULF range: GEOS-1 and2 results, Planet. Space Sci., 30, 1229, 1982.

Roux, A., S. Perraut, J.L. Rauch, C. de Villedary, G. Kremser, A. Korth, and D. T. Young, Wave-particle interaction near $\Omega_{\mathrm{He}^{+}}$observed on board GEOS 1 and 2, 2. Generation of ion cyclotron waves and heating of $\mathrm{He}^{+}$ions, J. Geophys. Res., 87, 8174, 1982.

Taylor, W. W. L., B. K. Parady, and L. J. Cahill, Explorer 45 observations of 1 - to $30-\mathrm{Hz}$ magnetic fields near plasmapause during magnetic storms, J. Geophys. Res., 80, 1271, 1975.

Young, D. T., S. Perraut, A. Roux, C. de Villedary, R. Gendrin, A. Korth, G. Kremser, and D. Jones, Wave-particle interactions near $\Omega_{\mathrm{He}^{+}}$observed on GEOS 1 and 2, 1. Propagation of ion cyclotron waves in the $\mathrm{He}^{+}$rich plasma, J. Geophys. Res., 86, 6755, 1981.

G. V. Khazanov, T. E. Moore, and M. W. Liemohn, Space Sciences Laboratory, NASA/MSFC, ES-83, Huntsville, AL 35812 (khazanov@mpb.msfc.nasa.gov).

E. N. Krivorutsky and J. L. Horwitz, Center for Space Plasma and Aeronomic Research. The University of Alabama in Huntsville, Huntsville, AL 35899 (horwitzj@ cspar.uah.edu).

Received: November 6, 1995; revised: February 23, 1996; accepted: February 29, 1996. 